\title{
Manifestaciones urológicas del pseudoxantoma elástico: A propósito de un caso
}

\author{
M. Angel Ortiz Gorraiz, A. Casares Arias, M. Tallada Buñuel, FJ Vicente Prados, \\ B. Honrubia Vílchez, A. Fernández Sánchez
}

Servicio de Urología. Hospital Universitario Virgen de las Nieves. Granada. España.

Actas Urol Esp 2005; 29 (1): 96-99

\section{RESUMEN}

MANIFESTACIONES UROLÓGICAS DEL PSEUDOXANTOMA ELÁSTICO: A PROPÓSITO DE UN CASO

Caso clínico: Mujer de 21 años con estrías angioides retinianas y estrías cutáneas en axilas y cuello. Tras biopsia cutánea con calcificaciones en las fibras elásticas de dermis, fue diagnosticada de Pseudoxantoma elástico. Remitida al Servicio de Urología por infección del tracto urinario inferior, en estudio ecográfico renal presentó múltiples focos hiperecogénicos de pequeño tamaño en la unión córtico-medular.

Comentarios: El Pseudoxantoma elástico es una rara enfermedad genética que se caracteriza por la fragmentación y calcificación de las fibras elásticas de la piel y arterias. Las manifestaciones clínicas incluyen hipertensión, angor pectoris, ictus, claudicación intermitente, hemorragia digestiva alta, estrías angioides retinianas y estrías cutáneas. La ecografia se caracteriza por la presencia de múltiples focos hiperecogénicos a nivel del parénquima renal, pero este hallazgo no es específico del Pseudoxantoma elástico. Sin embargo, la presencia de este patrón ultrasonográfico en pacientes jóvenes con anomalías dermatológicas debe hacernos pensar en este sindrome dentro de las diferentes posibilidades diagnósticas. Aparte de los hallazgos ecográficos descritos, sólo una lesión ureteral en una paciente sometida a ureteroscopia ha sido descrita en relación al ámbito urológico de este síndrome. En nuestro caso, la relación entre esta enfermedad y la presencia de infección urinaria recurrente no esta clara.

Palabras clave: Pseudoxantoma elástico. Ecografía. Infección urinaria.

\section{ABSTRACT \\ UROLOGIC FINDINGS IN PSEUDOXANTHOMA ELASTICUM: REPORT ONE CASE}

Case report: A 21-year-old woman presented with retinal angioid streaks and yellowish streak skin abnormalities in neck and axillary folds. Skin biopsy showed bluish-gray tangled masses of calcified elastic fibers in the mid-to lower dermis suggestive of Pseudoxanthoma elasticum (PXE). She consulted in Urology Department for lower urinary tract infection. Renal ultrasonography revealed multiple highly reflective foci in the corticomedullary junction.

Comment: Pseudoxanthoma elasticum is a rare genetic disorder characterised by fragmentation and calcification of elastic fibers in the skin and media of arteries. Frequent manifestations include hypertension, angina pectoris, transient cerebral ischemic attacks, intermittent claudication, upper gastrointestinal bledding, retinal angioid streaks and thickened skin. A characteristic appearance of highly reflective foci in the renal parenchyma have been reported in patients with PXE, but it's not specific for this syndrome. However, the presence of this structural pattern in a young patient with dermatological abnormalities should lead to the consideration of PXE in the differential diagnosis list. Besides ultrasonography findings, only a ureteral disruption case in a patient underwent ureteroscopic manipulation has been described to our knowledge. In our case, the true significance of these disease in recurrent urinary tract infection is uncertain.

Key words: Pseudoxanthoma elasticum. Ultrasonography. Urinary infection..
\end{abstract}

$\mathrm{E}$ 1 sindrome de Gronblad-Strandberg o Pseudoxantoma Elástico (PXE) es una enfermedad hereditaria del tejido conjuntivo en el que las fibras elásticas de la piel, retina y aparato cardiovascular fundamentalmente se calcifican, produciendo lesiones características ${ }^{1}$. Fue descrito por primera vez hace unos cien años, como un cuadro de lesiones dermatológicas que se pensaba eran depósitos de colesterol llamados xantomas.

Se estima una frecuencia de 1/100.000 individuos. Generalmente se diagnostica en el adulto joven en la $2^{\mathrm{a}}$ y $3^{\mathrm{a}}$ décadas, siendo $2 \asymp 5$ veces más frecuente en el sexo femenino ${ }^{1}$. 
Se trata de una enfermedad de etiología desconocida, en la que se implican factores genéticos con herencia autosómica recesiva, aunque se han descrito formas autosómicas dominantes. El gen defectuoso se localiza en el brazo corto del cromosoma 16 (16p 13.1) $)^{2}$. Recientes estudios han relacionado esta enfermedad con un déficit en ciertos tejidos de una proteína de transporte transmembrana, denominada ABCC6, y cuya baja expresión motivaría una falta de sustratos de la matriz extracelular, con perdida en el recambio necesario del tejido conectivo ${ }^{3-5}$.

Clínicamente presenta una gran heterogeneicidad de unos pacientes a otros, dependiendo del número de órganos implicados, y de su grado de afectación. Las primeras manifestaciones clínicas son frecuentemente las dermatológicas, que aparecen como pequeñas placas amarillentas y de consistencia laxa en áreas de flexión: cuello, axilas y región inguinal. Suelen progresar durante la infancia y adolescencia, estabilizándose en la edad adulta. A nivel ocular son patognomónicas las estrías angiodes, secundarias a la rotura de la membrana elástica de Burch, y son responsables de la ceguera producto de la esclerosis de la retina y degeneración macular. Se acompañan de hemorragias retinianas. En el aparato cardiovascular se produce degeneración del tejido conjuntivo de la túnica media de los vasos, válvulas y miocardio, pero respetando los vasos coronarios. Estos cambios provocan calcificaciones de los vasos, con signos de arteriopatía como son la claudicación intermitente, angor e hipertensión en el $15 \%$ de los casos, así como hemorragias a distintos niveles, pero sobre todo gastrointestinales hasta en un $10 \%$ de los pacientes ${ }^{6}$.

El diagnóstico de confirmación se realiza mediante biopsia de la piel o de paredes vasculares, detectándose en el microscopio óptico depósitos de calcio sobre las fibras elásticas ${ }^{6}$.

No existe un tratamiento curativo para el PXE. El láser tiene resultados poco eficaces en tratar la degeneración macular. Las hemorragias retinianas se previenen con suplementos vitamínicos de tipo A, C, E, así como de Zinc o Selenio. La cirugía plástica para las lesiones cutáneas y la cirugía cardiaca para las valvulopatías son otras opciones de tratamiento ${ }^{1}$.
Desde el punto de vista urológico, poco se sabe sobre las manifestaciones del PXE sobre el aparato genitourinario.

\section{CASO CLÍNICO}

Paciente mujer de 21 años diagnosticada hace 6 meses de estrías angioides de retina así como de estrías amarillentas en piel de cuello y axila. La biopsia cutánea mostró depósitos de calcio sobre las fibras elásticas hinchadas, degeneradas y fragmentadas sobre todo a nivel de la capa dérmica media e inferior, todo esto sugestivo de Pseudoxantoma Elástico.

La paciente fue derivada al Servicio de Urología por presentar clínica persistente en el último año de infección del tracto urinario inferior, con disuria, polaquiuria, estranguria y dolor suprapúbico. El hemograma y bioquímica de sangre estaban en rangos de normalidad, con buena función renal. El sedimento urinario reveló piuria con microhematuria. La infección urinaria fue confirmada mediante urocultivo, que presentó positividad para E. coli. Tras tratamiento antibiótico especifico la paciente mejoró temporalmente, con nuevo cultivo positivo para E. coli a los 2 meses, decidiéndose iniciar el estudio urológico mediante ecografía renal y vesical. A nivel renal se demostró la presencia de múltiples focos hiperrefringentes de pocos milímetros que no dejaban sombra posterior, fundamentalmente distribuidos en la unión corticomedular (Figura 1 y 2). No aparecieron hallazgos significativos a nivel vesical.

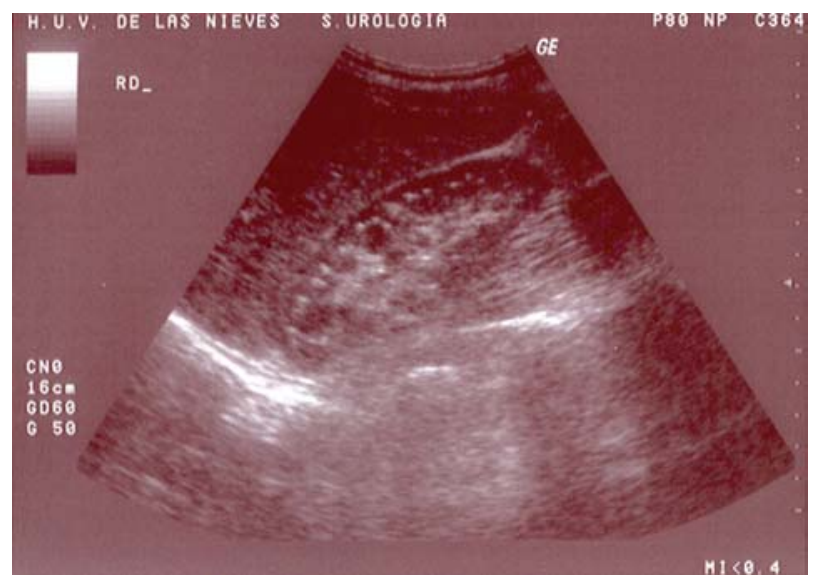

Figura 1. Ecografia renal, corte longitudinal, que mostraba múltiples focos hiperecogénicos sin sombra posterior a nivel del parénquima. 


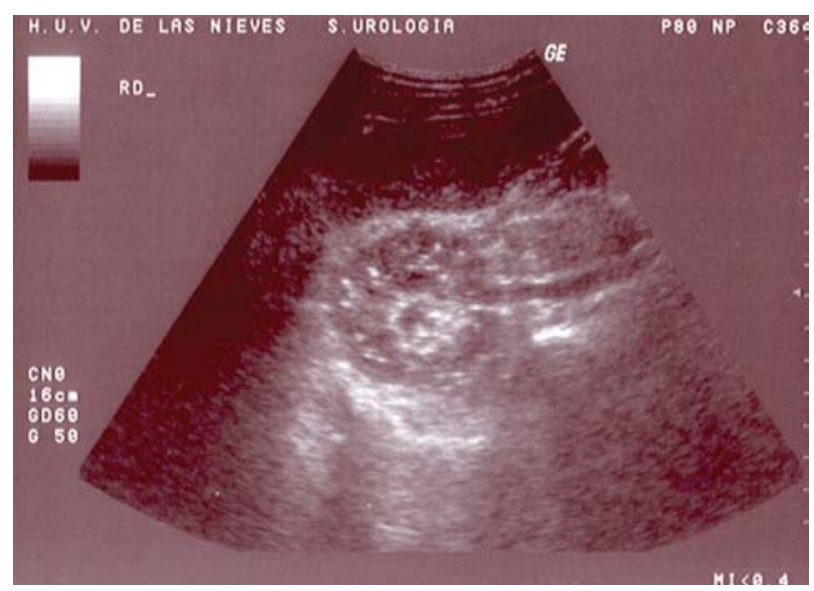

Figura 2. Mismo riñón en corte transversal que muestra igualmente numerosas imágenes hiperrefringentes a nivel de la unión córticomedular renal.

Actualmente la paciente sigue un control multidisciplinar, con controles periódicos por parte de Urología por sus infecciones de repetición.

\section{DISCUSIÓN}

El Pseudoxantoma Elástico es un raro desorden genético caracterizado por la fragmentación y calcificación de las fibras elásticas de la piel y túnica media de las arterias. Sus manifestaciones clínicas incluyen hipertensión, angor pectoris, accidentes isquémicos transitorios cerebrales, claudicación intermitente, sangrado gastrointestinal, dolor abdominal intermitente, estrías angioides retinales y estrías cutáneas. No existe un tratamiento especifico para el PXE y el manejo va encaminado hacia un diagnóstico precoz con un tratamiento anticipado de las posibles complicaciones ${ }^{1}$.

La aparición ecográfica de múltiples focos hiperecogénicos en el parénquima renal ha sido descrito en pacientes afectos de PXE, pero no es especifico para este síndrome ${ }^{7}$. Sin embargo, la presencia de este patrón ecográfico en un paciente joven con alteraciones cutáneas debe llevarnos a considerar el PXE dentro de los posibles diagnósticos diferenciales.

En el caso descrito, se llegó al diagnóstico de PXE mediante la realización de biopsia cutánea, siendo esta la técnica más idónea tal y como se describe en otros casos de la literatura ${ }^{3}$. La aparición de múltiples focos hiperecogénicos a nivel renal en el estudio ecográfico, patrón igualmente descrito en otros casos de PXE ya publicados ${ }^{7,8}$, nos lleva a pensar que se trata de una asociación clara entre ambos, puesto que el fenómeno de la calcifilaxis, descrito en pacientes con este síndrome $^{6}$, y por el cual existe una distribución generalizada de calcificaciones a nivel de los tejidos y de los vasos sanguíneos, explicaría el porqué de la presencia de este hallazgo ecográfico en una paciente con PXE, sin necesidad de confirmación histopatológica.

Hallazgos sonográficos similares han sido descritos tambien en pacientes con hipercalciuria; calcionosis $^{8}$; hipervitaminosis D; en hiperparatiroidismo $1^{\circ}$ y $2^{\circ}$; infección oportunistas renales en pacientes con SIDA por Pneumocystis carinii, Mycobacterium avium-intracellulare y citomegalovirus $^{9}$; en riñones trasplantados con rechazo crónico y en niños con hipertensión arterial secundaria a enfermedad de pequeño vaso. $Y$ por otro lado, en pacientes con PXE han aparecido focos hiperecogénicos en otras vísceras como páncreas y bazo ${ }^{10}$.

La apariencia ecográfica encontrada en los pacientes con PXE se ha relacionado con un aumento en la reflectividad de las arterias interlobares y arcuatas del riñón secundaria al deposito de calcio, idea con la cual estamos de acuerdo. En algunos estudios mediante ecoDoppler no se ha demostrado un descenso del flujo sanguíneo a nivel de la zona afectada por estos múltiples focos hiperecogénicos ${ }^{11}$. Sin embargo algunos autores han descrito como la degeneración de las fibras elásticas de la túnica media de las arterias conduce a una calcificación y fragmentación de éstas, con una subsiguiente proliferación de la intima causando una obstrucción de la luz vascular ${ }^{12}$. Estos cambios estarian asociados con una aterosclerosis temprana que motivaría la aparición de claudicación intermitente, hipertensión renovascular, dolor abdominal recurrente y angor pectoris.

El PXE no ha tenido una especial relevancia en el ámbito de la Urología. Además de los hallazgos ecográficos descritos, hemos encontrado en la revisión bibliográfica realizada un caso de lesión ureteral en un paciente diagnosticado de este síndrome y sometido a ureteroscopia por una litiasis ureteral $^{13}$. Los autores comentan en este articulo que la falta de elasticidad ure- 
teral secundaria al PXE pudo ser un factor precipitante de la lesión ureteral tras la manipulación ureteroscópica, recomendando un especial manejo de las presiones en estos pacientes.

Otras manifestaciones nefrourológicas descritas han sido la presencia de hipertensión renovascular, relacionado con el aumento de la resistencia vascular intrarrenal secundaria a la obstrucción progresiva de la luz de los vasos renales ${ }^{12}$. También se han descrito lesiones cutáneas con biopsia diagnostica para PXE en pacientes con insuficiencia renal crónica terminal de causa incierta sometidos a hemodiálisis durante largos periodos de tiempo ${ }^{14}$. Se han asociado igualmente la aparición de quistes a nivel renal y adrenal en estos pacientes ${ }^{15}$

En cuanto a las infecciones urinarias bajas de repetición que refería la paciente por nosotros presentada, creemos que se trata de un cuadro de cistitis recurrente habitual en pacientes jóvenes de sexo femenino, sin clara relación con el PXE que la paciente sufría, y cuya asociación por ahora no había sido descrita.

\section{REFERENCIAS}

1. Viljoen D. Pseudoxanthoma elasticum (Gronblad-Strandberg syndrome). J Med Genet 1988; 25: 488-490.

2. Struk B, Neldner KH, Rao VS, ST Jean P, Lindpaintner K. Mapping of both autosomal recessive and dominants variants of pseudoxanthoma elasticum to chromosome 16p13.1. Hum Molec Genet 1997; 6: 1823-1828.

3. HU X, Plomp AS, Van Soest S, et al. Pseudoxanthoma elasticum: a clinical, histopathological, and molecular update. Surv Ophthalmol 2003 Jul; 48(4): 424-438.

4. Bergen AA, Plomp AS, Schuurman EJ, et al. Mutation in ABCC6 cause pseudoxanthoma elasticum. Nature Genet 2000; 25: 228-231.

5. Ringpfeil F, Lebwohl MG, Christiano AM, Uitto J. Pseudoxanthoma elasticum: mutations in the MRP6 gene encoding a transmembrane ATP-binding casette (ABC) transporter. Proc Nat Acad Sci 2000; 97: 6001-6006.

6. Nikko AP, Dunningan M, Cockerell CJ. Calciphylaxis with histologic changes of pseudoxanthoma elasticum. Am J Dermatopathol 1996 Aug; 18(4): 396-399.

7. Domjan JM, Dewbury KC. Case report: multiple highly reflective foci in the renal parenchyma are not specific for pseudoxanthoma elasticum. Br J Radiol 1996 Sep; 69 (825): 871-872.

8. Gresser U, Stautner-Bruckmann C, Zoller WG. Kidney involvement in pseudoxanthoma elasticum sonography shows early calcinosis of the kidney parenchyma. Bildgebung 1987-89; 56(5): 179-180.

9. Dunnick NR, Sandler CM, Newhouse JH, Amis ES. Renal inflamatory disease. En: Textbook of Uroradiology, 3rd edition. Editor Barry B. Editorial Lippincott Williams \& Wilkins 2001: 150-177. Philadelphia.

10. Suarez MJ, Garcia JB, Orense M. Raimunde E, Lopez MV, Fernández O. Sonographic aspects of pseudoxanthoma elasticum. Pediatr Radiol 1991; 21(7): 538-539.

11. Crespi G, Derchi LE, Saffioti S. Sonographic detection of renal changes in pseudoxanthoma elasticum. Urol Radiol 1992; 13(4): 223-225.

12. Belli A, Cawthorne S. Visceral angiographic findings in pseudoxanthoma elasticum. Br J Radiol 1988 May; 61 (725): 368-371.

13. Heaton JP', Wilson JW. Pseudoxanthoma elasticum and its urological implications. J Urol 1986 Apr; 135(4): 776777.

14. Nickoloff BJ, Noodleman FR, Abel EA. Perforating pseudoxanthoma elasticum associated with chronic renal failure and hemodialysis. Arch Dermatol 1985 Oct; 121(10): 1321-1322.

15. Rossi A, Uzzauto MT. Pseudoxanthoma elasticum associated with endocrine disorders, renal and adrenal cystic dysplassia, sensorineural hearing loss and mental disorders. G Ital Dermatol Venereol 1990 Dec; 125(12): 593599.

Manuel Angel Ortiz Gorraiz

C/ Don Quijote 3, $3^{\circ}$ H. C.P. 18008 Granada

(Trabajo recibido el 8 julio de 2004) 